\title{
Adult mesenchymal stem cell ageing interplays with depressed mitochondrial Ndufs6
}

\author{
Yuelin Zhang 1,2, Liyan Guo ${ }^{2}$, Shuo Han ${ }^{3}$, Ling Chen ${ }^{3}$, Cheng Li ${ }^{3}$, Zhao Zhang ${ }^{2,3}$, Yimei Hong ${ }^{1}$, Xiaoxian Zhang ${ }^{2}$, \\ Xiaoya Zhou ${ }^{2}$, Dan Jiang ${ }^{3}$, Xiaoting Liang ${ }^{4}$, Jianxiang Qiu², Jinqiu Zhang ${ }^{2}$, Xin Li' ${ }^{1}$, Shilong Zhong ${ }^{5}$, Can Liao², Bin Yan' \\ Hung-Fat Tse ${ }^{3}$ and Qizhou Lian (10) 2,3
}

\begin{abstract}
Mesenchymal stem cell (MSC)-based therapy has emerged as a novel strategy to treat many degenerative diseases. Accumulating evidence shows that the function of MSCs declines with age, thus limiting their regenerative capacity. Nonetheless, the underlying mechanisms that control MSC ageing are not well understood. We show that compared with bone marrow-MSCs (BM-MSCs) isolated from young and aged samples, NADH dehydrogenase (ubiquinone) ironsulfur protein 6 (Ndufs6) is depressed in aged MSCs. Similar to that of Ndufs6 knockout (Ndufs6 ${ }^{-1-}$ ) mice, MSCs exhibited a reduced self-renewal and differentiation capacity with a tendency to senescence in the presence of an increased p53/p21 level. Downregulation of Ndufs6 by siRNA also accelerated progression of wild-type BM-MSCs to an aged state. In contrast, replenishment of Ndufs6 in Ndufs $6^{-1-}$-BM-MSCs significantly rejuvenated senescent cells and restored their proliferative ability. Compared with BM-MSCs, Ndufs6 ${ }^{-1-}-\mathrm{BM}-\mathrm{MSC}$ displayed increased intracellular and mitochondrial reactive oxygen species (ROS), and decreased mitochondrial membrane potential. Treatment of Ndufs $6^{-1-}$-BM-MSCs with mitochondrial ROS inhibitor Mito-TEMPO notably reversed the cellular senescence and reduced the increased p53/p21 level. We provide direct evidence that impairment of mitochondrial Ndufs6 is a putative accelerator of adult stem cell ageing that is associated with excessive ROS accumulation and upregulation of p53/p21. It also indicates that manipulation of mitochondrial function is critical and can effectively protect adult stem cells against senescence.
\end{abstract}

\section{Introduction}

Preclinical and clinical trials have revealed mesenchymal stem cell (MSC)-based therapy to be a promising therapeutic strategy for many diseases ${ }^{1-5}$. Compared with other types of stem cells currently under investigation, MSCs have several appreciable advantages, including easy isolation and being highly expandable with multilineage differentiation potential and low immunogenicity. Nonetheless an increasing body of evidence has demonstrated

\footnotetext{
Correspondence: Qizhou Lian (qzlian@hku.hk)

'Department of Emergency Medicine, Guangdong Provincial People's Hospital, Guangdong Academy of Medical Sciences, Guangzhou, Guangdong, China

${ }^{2}$ Guangzhou Women and Children's Medical Centre, Guangzhou Medical University, Guangzhou, Guangdong, China

Full list of author information is available at the end of the article

These authors contributed equally: Yuelin Zhang, Liyan Guo

Edited by Y. Shi
}

that their benefits decline during the aging process. Prolonged ex vivo cell culture of MSCs isolated from ageing donors show a senescent state, and reduced proliferation and impaired differentiation capacity, thus limiting their clinical application ${ }^{6,7}$ although the molecular network governing this senescence largely remains elusive. Exploration of the mechanisms that underlie cellular senescence is urgently needed.

To date, several potential mechanisms, including telomere shortening ${ }^{8}$, impaired autophagy ${ }^{9}$, and increased reactive oxygen species (ROS) have been reported to mediate the senescence of MSCs. Importantly, in addition to these factors stem cell senescence is strongly associated with mitochondrial dysfunction. One theory is that dysfunctional mitochondria accumulate with age ${ }^{10}$, while another proposes that mitochondrial dysfunction can

\section{(c) The Author(s) 2020}

(c) (i) Open Access This article is licensed under a Creative Commons Attribution 4.0 International License, which permits use, sharing, adaptation, distribution and reproduction cc) in any medium or format, as long as you give appropriate credit to the original author(s) and the source, provide a link to the Creative Commons license, and indicate if changes were made. The images or other third party material in this article are included in the article's Creative Commons license, unless indicated otherwise in a credit line to the material. If material is not included in the article's Creative Commons license and your intended use is not permitted by statutory regulation or exceeds the permitted use, you will need to obtain permission directly from the copyright holder. To view a copy of this license, visit http://creativecommons.org/licenses/by/4.0/. 
directly induce stem cell ageing and impair autophagic function with a consequent decline in their regenerative function ${ }^{11,12}$. Mitochondria can no longer be viewed as simple bioenergy factories, but rather as platforms for intracellular signaling, regulators of innate immunity, and modulators of stem cell activity. In turn, each of these properties provides clues as to how mitochondria might regulate aging and age-related diseases ${ }^{13}$. Compared with young MSCs, senescent MSCs exhibit increased ROS, largely as a result of mitochondrial structural remodeling $^{14}$. Inflammation-induced ROS leads to MSC senescence by upregulating the expression of miR-155 that in turn suppresses the expression of redox genes including Nfe2l2, Sod1, and Hmox $1^{15}$. Moreover, cholesterol reduces senescence in bone marrow-MSCs (BM-MSCs) by inhibiting the ROS/p53/p21 ${ }^{\text {Cip1/Waf1 }}$ pathway, indicating that ROS plays a very important role in the senescence of $\mathrm{MSCs}^{16}$. To the best of our knowledge, mitochondria are the major source of ROS and structural alterations to mitochondria result in their excessive level ${ }^{17}$.

Mitochondrial dysfunction with consequent increased ROS level is closely related to cellular senescence ${ }^{18,19}$. Among the mitochondrial complexes in the electron transport chains, mitochondrial complex I is most commonly impaired in aged $\mathrm{MSCs}^{20,21}$. It is the largest component of the mitochondrial respiratory chain and the major entry point for electrons into the process of oxidative phosphorylation (OXPHOS). The OXPHOS machinery in mitochondria has five complexes, including complex I, complex II (succinate dehydrogenase), complex III (cytochrome bc1 complex), complex IV (cytochrome c oxidase, COX), and complex V (ATP synthase $)^{22}$. These complexes are localized within the inner mitochondrial membrane. Complex I mediates ROS generation, and complex I defect leads to increased ROS production and decreased antioxidant defenses, thus impairing mitochondrial function ${ }^{23-25}$. Although complex I consists of at least 45 subunits, little is known about which subunit contributes to its stability and activity in MSC ageing.

NADH dehydrogenase (ubiquinone) iron-sulfur protein 6 (Ndufs6) is one of the major accessory subunits of complex I. Loss of this subunit caused by mutations has been reported to lead to fetal disease via mitochondrial complex I deficiency ${ }^{26}$. A previous study also demonstrated that Ndufs6 knockdown resulted in complex I instability and functional deficiency, leading to renal impairment due to increased ROS generation ${ }^{23}$. Given that Ndufs6 has been linked to complex I function, we hypothesized that Ndufs6 plays putative roles in MSC ageing and that Ndufs6 mediates cell senescence by regulating complex I function. Whether and how Ndufs6 mediates MSC senescence has nevertheless not been determined. In this study, we reveal that Ndufs6 plays a critical role in the regulation of BM-MSC senescence, and downregulation of Ndufs6 accelerates BM-MSC senescence via complex I deficiency with consequent increased ROS generation and activation of the p53/p21 signaling pathway.

\section{Materials and methods \\ mRNA expression analysis}

Five public mRNA expression microarray datasets were utilized to identify mRNA expression changes to mRNA of Ndufs1-8 genes during the aging process in humans and mice. They included (1) GSE9593 (GEO accession number) representing early and senescent passages of human BM-derived MSCs. (2) GSE35959 representing human MSC aging and primary osteoporosis. (3) GSE70376 comparing muscle stem cells (satellite cells) in homeostatic conditions and after cardiotoxin injury. (4) GSE47177 comparing quiescent satellite cells from mouse hindlimb muscle mouse uninjured young and old ones. (5) GSE56560 comparing young and senescent human MSCs from two donors. The processed data of these studies were extracted from GEO database. Expression data of Ndufs1-8 genes were selected for the linear regression analysis.

\section{Characterization of BM-MSCs and Ndufs $6^{-1-}$-BM-MSCs}

All animal experiments were approved by the Committee on the Use of Live Animals in Teaching and Research of the University of Hong Kong (CULATR 203871-16). Gene-Trap (Ndufs6 ${ }^{\text {gt/gt }}$ ) mice were kindly provided by Dr. David R. Thorburn's Lab (Murdoch Children's Research Institute, Australia) ${ }^{27}$. The gene-trap embryonic stem cell lines (AR01380) were derived from129/Ola strain. The chimeric mice were bred to $\mathrm{C} 57 \mathrm{BL} / 6$ background. All mice were produced in the mixed genetic background of C57BL/6 and 129/Ola. To acquire Ndufs6 ${ }^{-1-}$ (KO) and Ndufs $6^{+/+}$wild-type (WT) offspring, heterozygous parents were mated, and genotyping were characterized ${ }^{27}$. BM-MSCs and Ndufs $6^{-1-}$-BM-MSCs were isolated from WT and Ndufs $6^{-1-}$-mice (6-8 weeks old), as previously described $^{28}$. Briefly, the femurs and tibias of WT and KO mice were harvested, and then flushed with PBS using a 23-gauge syringe. After filtering with a 70-mm filter and BM cells were plated in culture dishes with complete DMEM medium containing DMEM/low glucose (Gibco), 10\% FBS (Life Technologies, 16000), $5 \mathrm{ng} / \mathrm{mL}$ epidermal growth factor (PeProTech, AF-100-15), $5 \mathrm{ng} / \mathrm{mL}$ fibroblast growth factor (PeProTech, 100-18B), $0.1 \mathrm{mM} \mathrm{2-}$ mercaptoethanol (Life Technologies, 21985023), Glutamax (Life Technologies, 35050061), NEAA (Life Technologies, 11140050), and penicillin-streptomycin (Life Technologies, 15140122) and then incubated at $37^{\circ} \mathrm{C}$ with $5 \% \mathrm{CO}_{2}$ in a humidified chamber. Three hours later, non-adherent cells were removed and the adherent cells 
were cultured in complete DMEM medium. Surface marker profiling and differentiation capacity of MSCs were examined, as previously reported ${ }^{29}$. Briefly, $1.5 \times 10^{5}$ MSCs were stained with the following antibodies: Sca-1 (eBioscience, 11-5981-82), CD34 (eBioscience, 11-0341-82), CD45 (eBioscience, 48-0451-82), CD90 (eBioscience, 11-0903-82), and CD105 (eBioscience, PA5-12511). Isotype-matched antibodies were used as negative control. Finally, data were analyzed by collecting 30,000 events on Beckman Coulter FC500 using CXP Analysis 2.0 software. The differentiation capacity of adipogenesis, chondrogenesis, and osteogenesis of BM-MSCs and Ndufs6 $6^{-1-}$-BM-MSCs at passages 3 and 4 was evaluated using the appropriate commercial kit (adipogenesis: Life Technologies, A1007001; chondrogenesis: Life Technologies, A1007101; osteogenesis: Life Technologies, A1007201) according to the protocol. The differentiation medium was changed every 3 days.

\section{RT-PCR}

Genes related to adipogenesis (LPL and Pparg), chondrogensis (Acan and Col2a1), and osteogenesis (Bglap and Alpl) were detected by real-time PCR (RT-PCR), as previously reported ${ }^{29}$. Total RNA was extracted using RNeasy kit (Qiagen, 74104). A total of $1 \mu \mathrm{g}$ mRNA was reversetranscribed using PrimeScript RT reagent kit (Takara, RR047A). The program for PCR was as follows: $95^{\circ} \mathrm{C}$ for $10 \mathrm{~min}, 40$ cycles of $95^{\circ} \mathrm{C}$ for $15 \mathrm{~s}$, and $60^{\circ} \mathrm{C}$ for $1 \mathrm{~min}$. The primers for mRNA were as follows: LPL (F: TAACTGCCACTTCAACCACA, R: AATCAGCGTCATCAGGAGA), Acan (F: CAAGAAATCGAATCCCCAAATC, R: ACTT AGTCCACCCCTCCTCACAT), Pparg (TTTCAAGGGT GCCAGTTTCG, R: GGTGGGACTTTCCTGCTAATAC AA), Col2a1 (F: CCGAGTGGAAGAGCGGAGAC, R: CA GTGGACAGTAGACGGAGGAAAG), Bglap (F: CCCAG ACCTAGCAGACACCATG, R: TGTTCACTACCTTA TTGCCCTCC), Alpl (F: TGGACGGTGAACGGGAAAA, R: AGCACAGCCAGTGGAAGCAG), Ndufs6 (F: GTAC GACGCGTGGGGTC, R: CGACACTTGAACCCCGAA AC), p53 (F: CCCCTGTCATCTTTTGTCCCT, R: AGCT GGCAGAATAGCTTATTGAG), p21 (F: CCTGGTGATG TCCGACCTG, R: CCATGAGCGCATCGCAATC), and $\beta$-actin (F: AGAGGGAAATCGTGCGTGAC, R: TGCTG GAAGGTGGACAGTGAG). The fold change for mRNA relative to $\beta$-actin was determined by the formula: $2^{-\Delta \Delta C t}$.

\section{SA- $\beta$-gal staining}

SA- $\beta$-gal staining was performed according to the manufacturer's instructions (Cell Signaling Technology). Briefly, after washing with PBS, MSCs were fixed with fixative solution for $15 \mathrm{~min}$ at room temperature and then stained with SA- $\beta$-gal staining solution at $37^{\circ} \mathrm{C}$ overnight. Senescent MSCs were stained blue and were photographed. The percentage was calculated from five different view fields of each sample in three independent experiments.

\section{Transmission electron microscope}

The mitochondrial morphology and autophagosomes of BM-MSCs and Ndufs6 ${ }^{-1-}$-BM-MSCs were evaluated by a transmission electron microscope (TEM), as previously described $^{30}$. The samples were photographed randomly under a TEM (Hitachi, H-7650). Finally, the mitochondrial length and number of autophagosomes were calculated for five different view fields of each group in three independent experiments.

\section{Immunofluorescence staining}

Different groups of MSCs were cultured in 24-well plates with glass coverslips and fixed with $4 \%$ PFA, permeabilized with $0.1 \%$ Triton- 100 , and then blocked by $5 \%$ bovine serum albumin. Subsequently, cells were incubated with rabbit anti-Ki-67 (Abcam, ab15580) at $4{ }^{\circ} \mathrm{C}$ overnight followed by fluorescent secondary antibody. Finally, the sample was mounted with DAPI, and ten random fields photographed. The percentage of Ki-67-positive cells was calculated.

\section{siRNA intervention}

To knockdown of Ndufs6 in MSCs, Ndufs6-siRNA (Santa Cruz, sc-149888) and control siRNA (Santa Cruz, sc-37007) were used to transfect MSCs with a Lipofectamine RNAiMAX Reagent Kit (Invitrogen, 13778-075) at a standardized MOI (multiplicity of infection) of 5 according to the protocol. After $72 \mathrm{~h}$, MSCs were harvested and the silencing efficiency was evaluated by western blotting.

\section{Western blotting}

The proteins of the different groups of MSCs were extracted and the concentration measured by Bradford. A total amount of $25 \mu \mathrm{g}$ protein of each sample was loaded, separated by SDS/PAGE, and then transferred to a PVDF membrane. The membrane was blocked with $5 \%$ fat-free milk in TBST and then incubated with the following antibodies: OXPHOS (Abcam, ab110413), Ndufs6 (GeneTex, GTX88043), p53 (Santa Cruz, SC-98), p21 (Santa Cruz, SC-271532), and GAPDH (Santa Cruz, SC-137179) at $4{ }^{\circ} \mathrm{C}$ overnight. After washing with TBST, the membrane was incubated with horseradish peroxidaseconjugated secondary antibodies (1:10,000; Santa Cruz) at room temperature for $1 \mathrm{~h}$ and then allowed to develop.

\section{Measurement of ROS}

Intracellular and mitochondrial ROS were measured by 2',7'-dichlorodihydrofluorescein diacetate (DCFH-DA) and Mito-Sox staining, respectively. Briefly, MSCs from different experimental groups were cultured in 24-well 
plates with glass coverslips. Then MSCs were incubated with $10 \mu \mathrm{M}$ DCFH-DA (Invitrogen, C369) or $5 \mu \mathrm{M}$ MitoSox (Invitrogen, M36008) for $10 \mathrm{~min}$ at $37^{\circ} \mathrm{C}$ in the dark. Finally, the sample was photographed randomly and fluorescence intensity calculated for five different view fields of each group in three independent experiments, using Image J software. Moreover, the ROS level was also examined by flow cytometry. Briefly, MSCs were grown to $80 \%$ confluence in $6-\mathrm{cm}$ culture dish, and incubated in $10 \mu \mathrm{M}$ DCFH-DA or $5 \mu \mathrm{M}$ Mito-Sox with fluorescenceactivated cell-sorting staining buffer at $37^{\circ} \mathrm{C}$ for $10 \mathrm{~min}$. After incubation, MSCs were washed twice with PBS and analyzed by flow cytometry (FC500; Beckman Coulter). A shift to the right indicates increased ROS levels. BMMSCs were treated with $10 \mathrm{mM} \mathrm{H}_{2} \mathrm{O}_{2}$ for $6 \mathrm{~h}$ for DCFHDA staining or $100 \mathrm{mM}$ antimycin A for $30 \mathrm{~min}$ for MitoSOX staining as positive control.

\section{TMRM staining}

Mitochondrial membrane potential (MMP) was detected by tetramethylrhodamine ethyl ester perchlorate (TMRM, Invitrogen, T668) according to the protocol. Briefly, MSCs from different experimental groups were cultured in 24-well plates with glass coverslips and then stained with $50 \mathrm{nM}$ TMRM for $10 \mathrm{~min}$. Finally, the sample was photographed randomly and fluorescence intensity calculated for five view fields of each group in three independent experiments, using Image J software. Moreover, we also analyzed the MMP with TMRM staining, using flow cytometry. BM-MSCs were treated with $10 \mu \mathrm{M}$ carbonyl cyanide- $p$-trifluoromethoxyphenylhydrazone (FCCP) for $1.5 \mathrm{~h}$ as positive control.

\section{AAV transfection}

The adeno-associated virus-Ndufs6 (AAV2-Ndufs6) plasmid was constructed as previously reported ${ }^{31}$. Briefly, the open reading frame of human Ndufs6 (NM_004553.4) was amplified and cloned into an AAV2 core vector (Fig. S1). After the Sanger sequencing, the AAV2-Ndufs plasmids were packaged for viral products (BioWit Technologies, China). Ndufs6 ${ }^{-1-}$-BM-MSCs were infected using AAV2-Ndufs6 virus with an MOI of 20,000. The empty AAV2-infected Ndufs6 ${ }^{-1-}$-BM-MSCs cells were used as negative control. The infection efficiency was confirmed by western blotting for Ndufs 6 at $72 \mathrm{~h}$ post infection.

\section{Oxygen consumption rate measurement}

The mitochondrial function of MSCs was evaluated by seahorse XFp analyzer as previously described (Agilent Technologies, RRID: SCR_013575) ${ }^{1}$. Briefly, four bioenergetics measurements were detected: (1) basal reparation-in assay medium containing $4.5 \mathrm{~g} / \mathrm{L}$ glucose; (2) inhibition of ATP synthase-using oligomycin $(1 \mu \mathrm{M})$, ATP synthesis turnover, and respiration driving proton leak; (3) maximal mitochondrial respiratory capacitypost treatment with FCCP $(2 \mu \mathrm{M})$; and (4) nonmitochondrial respiration-following treatment with complex I inhibitor, rotenone $(2 \mu \mathrm{M})$.

\section{Statistical analysis}

All values are expressed as mean \pm SD. Statistical tests were performed using Prism version 5.0c (GraphPad Software). Statistical significance was determined by independent-samples $T$ test between two groups or analysis of variance followed by Bonferroni test between more than two groups. A $p<0.05$ was considered significant.

\section{Results}

\section{Accessory subunit of mitochondrial complex I Ndufs6 is downregulated in aged MSCs}

To examine the relationship between accessory subunits of mitochondrial complex I and aging, we first extracted gene expression of Ndufs1-8 from five publicly available microarray datasets representing young versus old/senescent cells and tissues. The datasets included (1) GSE9593 (GEO accession number) representing early and senescent passages of human BM-derived MSCs. (2) GSE35959 representing human MSCs from young, aging, and primary osteoporosis. (3) GSE70376 representing muscle stem cells (satellite cells) in homeostatic conditions and regenerative cardiotoxin injury-stimulated muscle stem cells from aged mice. (4) GSE47177 compared quiescent muscle stem cells (satellite cells) from uninjured mice, young and old. (5) GSE56560 representing passage 9 human MSCs from two donors at days 2 and 7 of culture. In all five datasets, we observed a similar decrease in mRNA expression of all eight Ndufs subunits during the aging process (Fig. S2). In particular, expression of Ndufs3, 4, and 6 was consistently and prominently decreased. We further analyzed the difference between Ndufs6 mRNA from young and old/ senescent cells and tissues using linear regression. A scatter plot for mRNA expression (relative to young) of all Ndufs subunits of mitochondria complex assembly I is shown in Fig. 1A. The mRNA expression of Ndufs6 is significant different between young and old cells or tissues (Fig. 1B). Linear regression of mRNA expression in the young is larger than that of the old samples (Fig. 1C). RT-PCR results showed that the mRNA level of Ndufs6 was much lower in aged-BM-MSCs derived from mice than young-BM-MSCs (Fig. S3). Further, the protein level of Ndufs6 was greatly reduced in agedBM-MSCs derived from mice compared with youngBM-MSCs (Fig. 1D). This preliminary finding indicates that a dominant decline in expression of Ndufs6 may be a pivotal factor that provokes MSC ageing or a consequence in aged MSCs. 
A

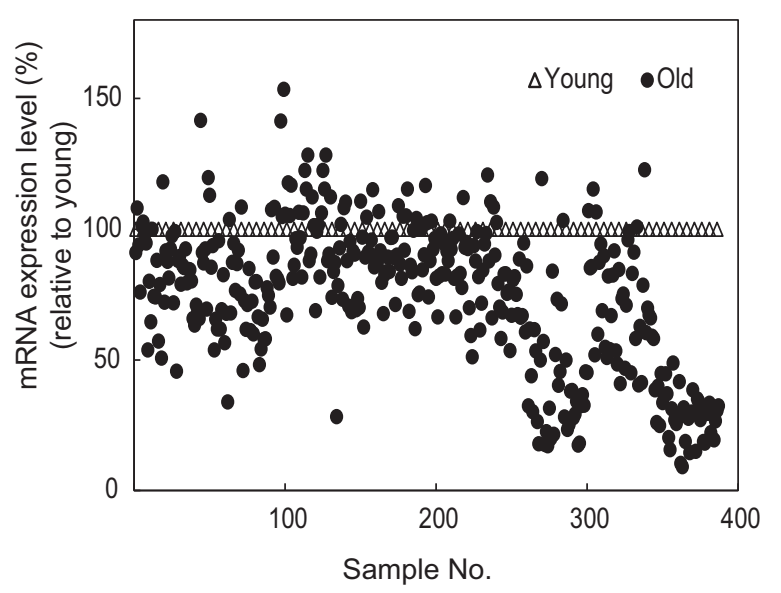

C

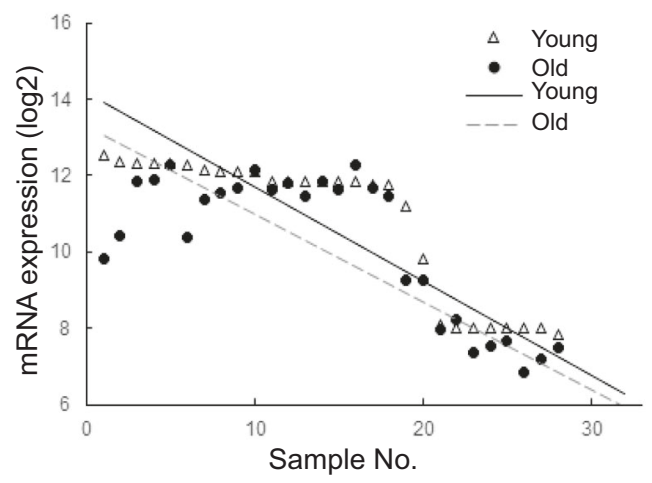

B

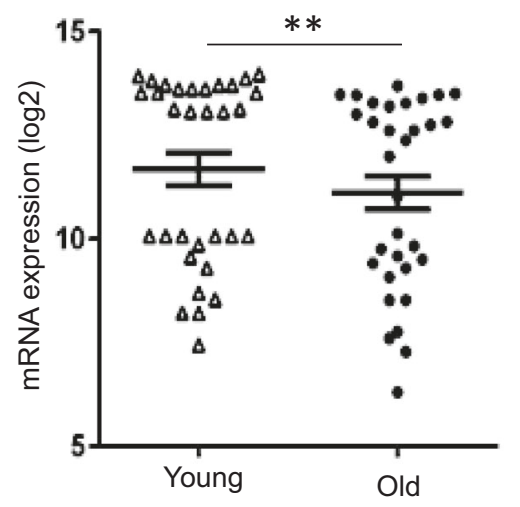

D
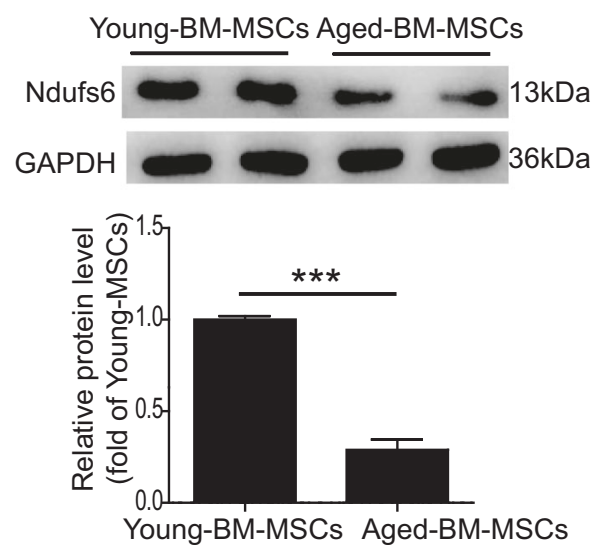

Fig. 1 mRNA expression change of Ndufs6 during aging in human or mouse MSCs. mRNA expression data were extracted from five microarray datasets representing young vs. old/senescent cells and tissues or early stages of MSC differentiation. A Scatter plot for mRNA expression (relative to young) for all subunits (Ndufs1-8) of mitochondria complex assembly I. B mRNA expression difference of Ndufs6 between young and old cells or tissues $(n=32)$. C Linear regression of mRNA expression of Ndufs6 between young and old cells or tissues $(n=30)$. D The protein level of Ndufs6 in young-BM-MSCs and aged-BM-MSCs isolated from mice was determined by western blotting. Significant difference between young and old cells or tissues, ${ }^{* *} p<0.01{ }^{* * *} p<0.001$.

\section{Reduced differentiation potential of BM-MSCs in the absence of Ndufs 6}

Given the important role of Ndufs6 in complex I activity and MSC ageing, we next characterized BM-MSCs from WT and Ndufs $6^{-1-}$ mice ${ }^{27}$. First, we examined the cell death between BM-MSCs and Ndufs6 ${ }^{-1-}$-BM-MSCs. Trypan blue staining showed that there was no difference of cell death between BM-MSCs and Ndufs6 ${ }^{-1-}$-BMMSCs (data not shown). Next, surface markers of BMMSCs and Ndufs6 $6^{-1-}$-BM-MSCs were examined by fluorescence cytometry. The results showed that BMMSCs and Ndufs6 ${ }^{-1-}$-BM-MSCs had similar surface markers, i.e., Sca (+), CD90 (+), CD105 (+), CD45 (-), and CD34 (-) (Fig. S4A). Western blotting showed that Ndufs6 was highly expressed in BM-MSCs, but not in
Ndufs $6^{-1-}$ BM-MSCs, confirming the absence of Ndufs 6 in Ndufs6 ${ }^{-1-}$-BM-MSCs (Fig. S4B). The differentiation capacity of BM-MSCs and Ndufs $6^{-1-}$-BM-MSCs for adipogenesis, chondrogenesis, and osteogenesis was evaluated by staining with oil red, alcian blue, and alizarin red, respectively. Compared with BM-MSCs, adipogenic, chondrogenic, and osteogenic differentiation capacity of Ndufs6 $6^{-1-}$-BM-MSCs was significantly reduced (Fig. S4C). To further verify their differentiation capacity, the genes related to adipogenesis (LPL and Pparg), chondrogenesis (Acan and Col2a1), and osteogenesis (Bglap and Alpl) were detected by RT-PCR. The mRNA expression of LPL, Pparg, Acan, Col2a1, Bglap, and Alpl was greatly decreased in Ndufs6 ${ }^{-1-}$-BM-MSCs in differentiation conditions compared with that of BM-MSCs 
(Fig. S4D-F). These results indicate that the differentiation functions of $\mathrm{Ndufs}^{-1-}$-BM-MSCs dramatically declined compared with WT BM-MSCs.

\section{Ndufs6 low-BM-MSCs exhibits increased cellular senescence}

Increasing evidence has shown that dysfunction of MSCs is caused by premature ageing of stem cells. We therefore compared the cellular senescence of BM-MSCs and Ndufs $6^{-l-}$-BM-MSCs. We first examined cellular proliferation by counting cell numbers of BM-MSCs and Ndufs6 $6^{-1-}$-BM-MSCs, and confirmed that the cellular proliferation of Ndufs6 ${ }^{-1-}$-BM-MSCs was significantly reduced (Fig. 2A). BM-MSCs exhibited a healthy spindle shape, whereas Ndufs6 $6^{-1-}$-BM-MSCs showed an enlarged and fried egg-like shape (Fig. 2B) with a notably increased cell area compared with BM-MSCs (Fig. 2C). Moreover, SA- $\beta$-gal staining showed that the number of SA- $\beta$-galpositive cells was greatly enhanced in $\mathrm{Ndufs}^{-1-}{ }^{-\mathrm{BM}-}$ MSCs compared with BM-MSCs (Fig. 2D, E). To further verify the senescence of Ndufs6 ${ }^{-1-}$-BM-MSCs, we performed Ki-67 staining to examine their proliferative capacity and showed that the of number Ki-67-positive cells was much lower in Ndufs6 ${ }^{-1-}$-BM-MSCs than BMMSCs (Fig. 2F, G). RT-PCR results showed that the mRNA level of p53 and p21, senescence-associated markers, was significantly elevated in $\mathrm{Ndufs}^{-1-}$-BM-MSCs compared with BM-MSCs (Fig. 2H). Moreover, western blotting analysis also showed that the protein level of p53 and $\mathrm{p} 21$ was much higher than BM-MSCs (Fig. 2I). These results suggest that Ndufs6 may play a critical role in regulating cellular senescence.

\section{Knockdown of Ndufs6 accelerates cellular senescence in BM-MSCs}

To further explore the relationship between Ndufs6 and cellular senescence, we performed experiments with lossand gain-of-function of Ndufs6 in MSCs. First, we used Ndufs6-siRNA to treat BM-MSCs. RT-PCR results showed that the mRNA level of Ndufs6 was markedly reduced, whereas the mRNA level of p53 and p21 was significantly enhanced in Ndufs6-siRNA-treated BMMSCs compared with BM-MSCs (Fig. 3A). Similarly, western blotting also showed that the protein level of Ndufs6 was markedly decreased in Ndufs6-siRNA-treated BM-MSCs compared with BM-MSCs (Fig. 3B). Further, the protein level of p53 and p21 was greatly upregulated compared with BM-MSCs (Fig. 3B). We then performed SA- $\beta$-gal staining to detect senescent MSCs among the two groups. Ndufs6-siRNA-treated BM-MSCs exhibited increased $\beta$-gal positivity compared with BM-MSCs (Fig. 3C, D). Moreover, Ki-67 staining demonstrated that the number of Ki-67-positive cells was significantly reduced in Ndufs6-siRNA-treated BM-MSCs (Fig. 3E, F).
Overall, these findings showed that knockdown of Ndufs6 enhanced cellular senescence of BM-MSCs.

\section{Replenishment of Ndufs 6 in Ndufs $6^{-/-}$-BM-MSCs largely reverses cellular senescence}

To restore Ndufs6 in Ndufs6 ${ }^{-1-}$-BM-MSCs, Ndufs6 ${ }^{-1-}$ -BM-MSCs were transduced with AAV containing Ndufs6. As expected, the mRNA level of p53 and p21 was much lower in Ndufs6-AAV-treated Ndufs $6^{-1-} \mathrm{BM}$ MSCs than control-AAV-treated Ndufs6 ${ }^{-1-}$-BM-MSCs (Fig. 4A). Western blotting showed that the protein level of Ndufs6 was notably enhanced, whereas the protein level of p53 and p21 was significantly downregulated in Ndufs6-AAV-treated Ndufs6 ${ }^{-1}-$-BM-MSCs compared with BM-MSCs (Fig. 4B). Next, we performed SA- $\beta$-gal staining to examine cellular senescence. As shown in Fig. $4 \mathrm{C}, \mathrm{D}$, the number of SA- $\beta$-gal-positive cells was dramatically reduced in Ndufs6 ${ }^{-1-}$-BM-MSCs with Ndufs6 replenishment compared with $\mathrm{Ndufs}^{-1-}{ }^{-\mathrm{BM}-}$ MSCs (Fig. 4C, D). The results were further confirmed by Ki-67 staining that revealed a significant increase of Ki-67 in Ndufs6 ${ }^{-1-}$-BM-MSCs with Ndufs6 overexpression (Fig. 4E, F). Furthermore, we also examined whether restoring Ndufs6 expression could revert the reduced differentiation capacity of Ndufs $6^{-1-}$-BM-MSCs. The differentiation capacity of $\mathrm{Ndufs}^{-1-}{ }^{-\mathrm{BM}-\mathrm{MSC} \text { a }}$ and Ndufs6 ${ }^{-1-}$-BM-MSCs with Ndufs6 overexpression for adipogenesis, chondrogenesis, and osteogenesis was evaluated by measuring the mRNA level of the genes related to adipogenesis (LPL and Pparg), chondrogenesis (Acan and Col2a1), and osteogenesis (Bglap and Alpl). As shown in Fig. S5, the mRNA expression of LPL, Pparg, Acan, Col2a1, Bglap, and Alpl was greatly increased in Ndufs6 ${ }^{-1-}$-BM-MSCs with Ndufs6 overexpression in differentiation conditions compared with that of Ndufs $6^{-1-}$-BM-MSCs, suggesting that restoring Ndufs6 expression could revert the reduced differentiation capacity of Ndufs6 $6^{-1-}$-BM-MSCs (Figure S5A-C). Taken together, these findings suggest that restoration of Ndufs6 in Ndufs6 $6^{-1-}$-BM-MSCs reduced cellular senescence.

\section{Loss of Ndufs6 results in complex I deficiency and enhanced ROS generation}

Since Ndufs6 is a major assembly subunit of complex I, absence of Ndufs6 may impair the activity of complex I. Western blotting results showed a reduced amount of complex I in Ndufs6 ${ }^{-1-}$-BM-MSCs compared with BMMSCs, presumably due to the absence of Ndufs6 (Fig. 5A). Nonetheless the protein level of complexes II, III, and IV did not differ significantly between the two (Fig. 5A), suggesting that knockout of Ndufs6 affects the function of complex I. Since complex I is closely linked to ROS generation, we subsequently examined ROS generation in Ndufs6 ${ }^{-1-}$-BM-MSCs. DCFH-DA staining showed that 


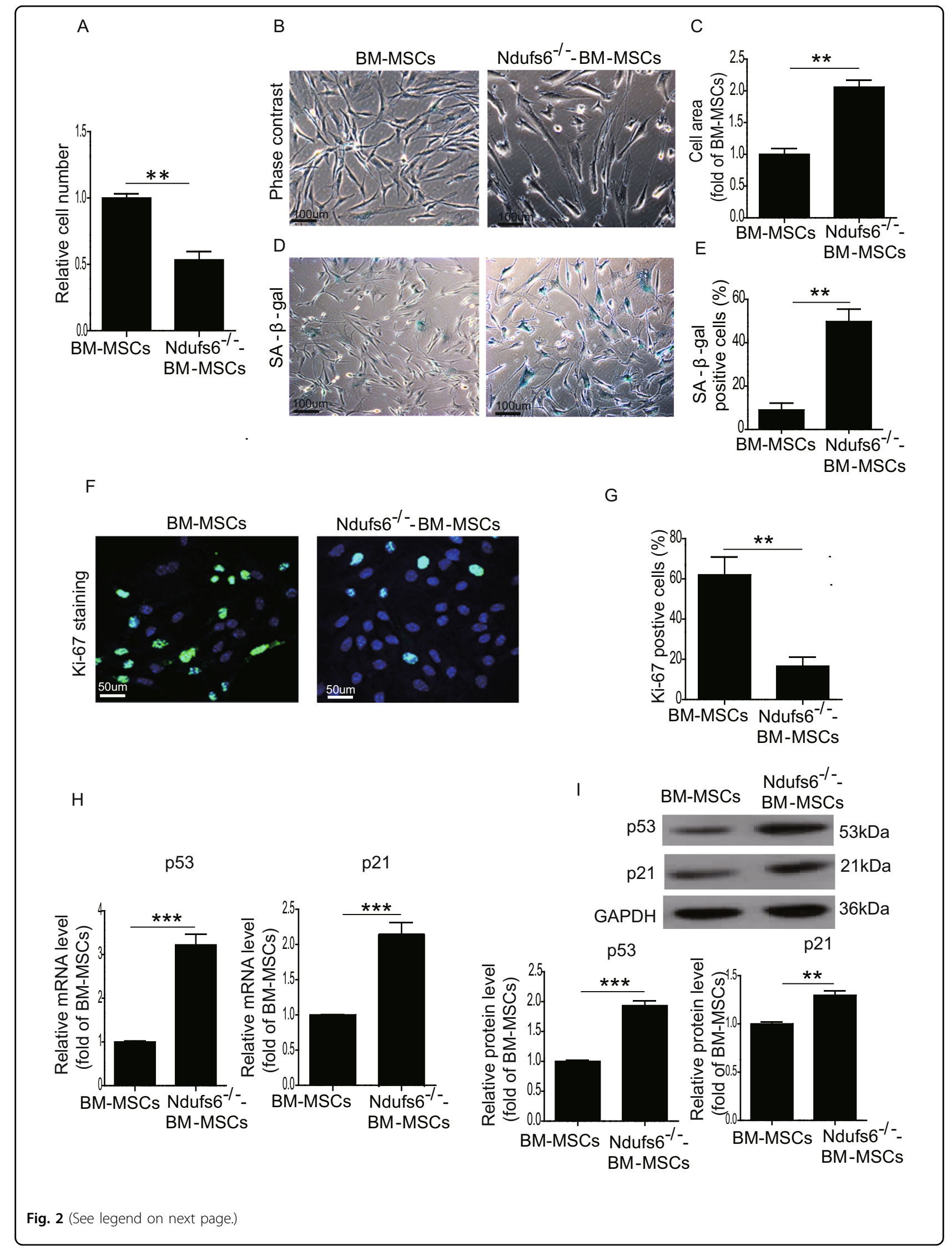


(see figure on previous page)

Fig. 2 Ndufs $6^{-1-}$-BM-MSCs exhibit increased cellular senescence. A The same number of BM-MSCs and Ndufs6 ${ }^{-1-}$-BM-MSCs was cultured, and cells counted and presented 2 days later. B The typical cellular morphology of BM-MSCs and Ndufs $6^{-1-}$-BM-MSCs was observed. C The cell area of

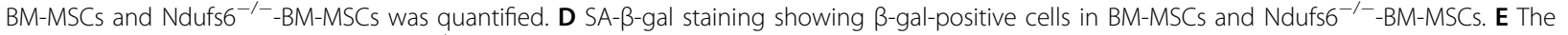
$\beta$-gal positivity in BM-MSCs and Ndufs $6^{-1-}$-BM-MSCs was quantified by calculating the ratio of stained cells to total cells. $\mathbf{F}$ The proliferation of BMMSCs and Ndufs6 ${ }^{-1-}$-BM-MSCs was assessed by Ki-67 staining. G The Ki-67 positivity in BM-MSCs and Ndufs6 ${ }^{-1-}-$ BM-MSCs was quantified by calculating the ratio of stained cells to total cells. $\mathbf{H}$ The mRNA level of p53 and p21 in BM-MSCs and Ndufs $6^{-1-}{ }_{-}^{-B M-M S C s}$ was determined by RT-PCR. I The protein level of p53 and p21 in BM-MSCs and Ndufs6 ${ }^{-1-}$-BM-MSCs was determined by western blotting. Results are presented as mean \pm SD. $n=3 .{ }^{* *} p<0.01 ;{ }^{* *} p<0.001$.

Ndufs6 ${ }^{-1-}$-BM-MSCs had an increased level of intracellular ROS compared with BM-MSCs (Fig. 5B, C). Flow cytometry analysis also showed a higher level of intracellular ROS in Ndufs6 ${ }^{-1-}$-BM-MSCs than BM-MSCs (Fig. S6A). Moreover, Mito-Sox staining demonstrated that the level of mitochondrial ROS was greatly enhanced in Ndufs6 ${ }^{-1-}$-BM-MSCs compared with BM-MSCs (Fig. 5D, E). Similarly, flow cytometry analysis also confirmed that the level of mitochondrial ROS was much higher in Ndufs6 ${ }^{-1-}$-BM-MSCs than BM-MSCs (Fig. S6B). Next, TMRM staining was employed to detect the MMP in MSCs. It showed a significantly reduced TMRM fluorescence in Ndufs6 ${ }^{-1-}$-BM-MSCs compared with BM-MSCs (Fig. 5F, G), indicating loss of MMP in Ndufs6 $6^{-1-}$-BM-MSCs. Consistently, flow cytometry analysis also confirmed that the level of MMP was much lower in $\mathrm{Ndufs}^{-1-}$-BM-MSCs than BM-MSCs (Fig. S6C). Overall, these results demonstrated that an absence of Ndufs 6 enhances ROS generation and damages mitochondria via complex I deficiency. Since complex I mediates mitochondrial function, we thus determined whether absence of Ndufs6 can influence mitochondrial morphology and autophagy in Ndufs6 $6^{-1-}$-BM-MSCs, using a TEM. As shown in Fig. S7, compared with BMMSCs, the mitochondrial length was greatly increased in Ndufs6 $6^{-1-}$-BM-MSCs (Fig. S7A, B). However, the number of autophagosomes in Ndufs6 ${ }^{-1-}$-BM-MSCs was much lower than that in BM-MSCs (Fig. S7A, C). Given that complex I deficiency could result in NADH accumulation, whether NAD supplementation could rescue Ndufs6 ${ }^{-1-}$-BM-MSC senescence has not been determined. Therefore, we treated the Ndufs $6^{-1-}$-BM-MSCs with $20 \mathrm{mM}$ nicotinamide riboside (NR) for $48 \mathrm{~h}$ and then assessed the cellular senescence. As shown in Fig. $5 \mathrm{H}$, compared with Ndufs6 ${ }^{-1-}$-BM-MSCs, the cellular senescence was greatly reduced in NR-treated Ndufs $6^{-1-}$ -BM-MSCs, indicating NR treatment could ameliorate senescence of Ndufs6-deficient MSCs (Fig. 5H).

\section{Downregulation of Ndufs6 induces cell senescence of MSCs via regulation of the ROS/p53 pathway}

To verify whether the absence of Ndufs6 induces cell senescence of MSCs via regulation of ROS, we used the mitochondrial ROS inhibitor Mito-TEMPO (Santa Cruz,
SC-221945, $100 \mu \mathrm{M})$ to treat $\mathrm{Ndufs}^{-/-}$-BM-MSCs for 48 hours. We first examined the cellular proliferation of Ndufs $6^{-1-}$-BM-MSCs and Mito-TEMPO-treated Ndufs $6^{-1-}$-BM-MSCs, and confirmed that the cellular proliferation of Mito-TEMPO-treated Ndufs6 ${ }^{-1-}$-BMMSCs was significantly increased (Fig. S8). Mito-TEMPOtreated Ndufs $6^{-1-}$-BM-MSCs exhibited a decreased level of mitochondrial ROS compared with $\mathrm{Ndufs}^{-1-}$-BMMSCs (Fig. 6A, B). SA- $\beta$-gal staining showed that the SA$\beta$-gal activity was dramatically reduced in Mito-TEMPOtreated Ndufs6 ${ }^{-1-}$-BM-MSCs compared with Ndufs6 ${ }^{-1-}$ -BM-MSCs (Fig. 6C, D). Furthermore, the number of Ki67-positive cells was significantly enhanced in MitoTEMPO-treated Ndufs6 ${ }^{-1-}$-BM-MSCs compared with Ndufs6 ${ }^{-1-}$-BM-MSCs (Fig. 6E, F). Subsequently, we compared the mRNA level of p53 and p21 in MitoTEMPO-treated Ndufs6 ${ }^{-1-}$-BM-MSCs and Ndufs $6^{-1-}$ -BM-MSCs, and found that the mRNA of p53 and p21 was much lower in Mito-TEMPO-treated Ndufs6 $6^{-1-}$-BMMSCs (Fig. 6G). Consistently, the protein level of p53 and p21 was also greatly downregulated in Mito-TEMPOtreated Ndufs6 ${ }^{-1-}$-BM-MSCs compared with BM-MSCs (Fig. 6h). To further evaluate the effects of Mito-TEMPO, we detected oxygen consumption rate (OCR) of BMMSCs, Ndufs6 ${ }^{-1-}$-BM-MSCs, and Mito-TEMPO-treated Ndufs6 ${ }^{-1-}$-BM-MSCs, using an extracellular flux analyzer (Fig. S9A). Compared with BM-MSCs, basal mitochondrial OCR and ATP production significantly reduced in Ndufs6 ${ }^{-1-}$-BM-MSCs (Fig. S9B, C). However, basal mitochondrial OCR and ATP production were markedly improved in $\mathrm{Ndufs}^{-/-}$-BM-MSCs with Mito-TEMPO treatment compared with Ndufs6 $6^{-1-}$-BM-MSCs (Fig. S9B, C). These results demonstrate that inhibition of mitochondrial ROS alleviated cell senescence of Ndufs $6^{-/-}$ -BM-MSCs, indicating that cellular senescence in MSCs due to Ndufs6 is associated with the ROS/p53/ p21 signaling pathway.

\section{Discussion}

There are several major findings of this study. First, we have determined that a mitochondrial complex I accessory subunit, Ndufs6, is notably downregulated in both human and mice-aged MSCs. Second, we have verified that downregulation of Ndufs6 is not only a consequence 

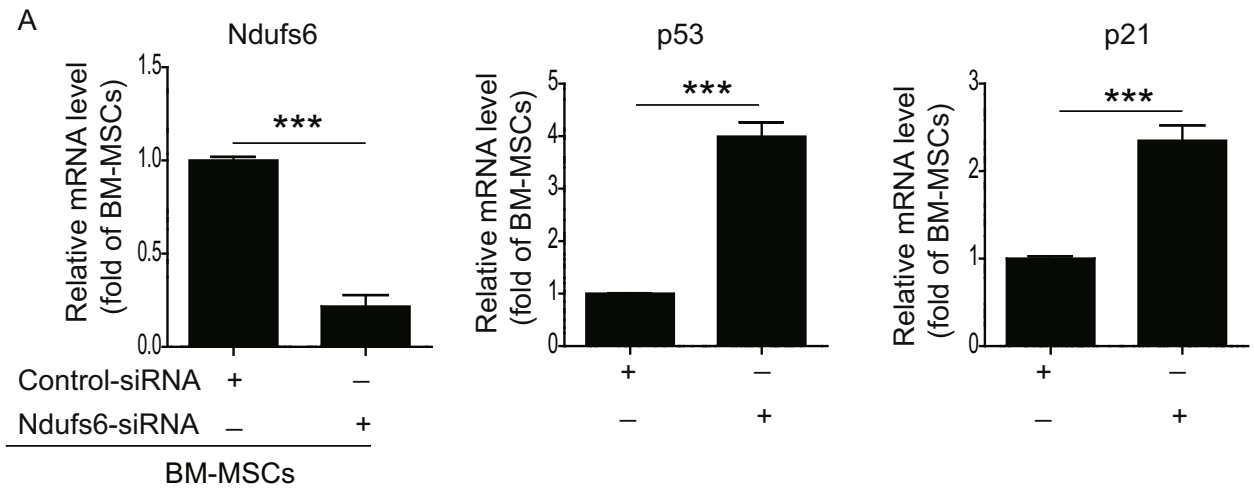

B
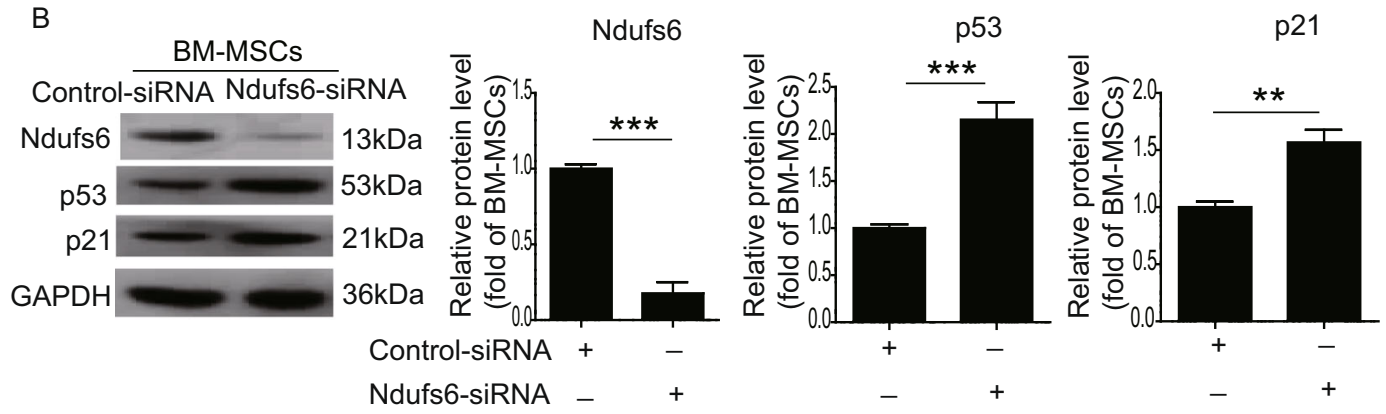

C

BM-MSCs
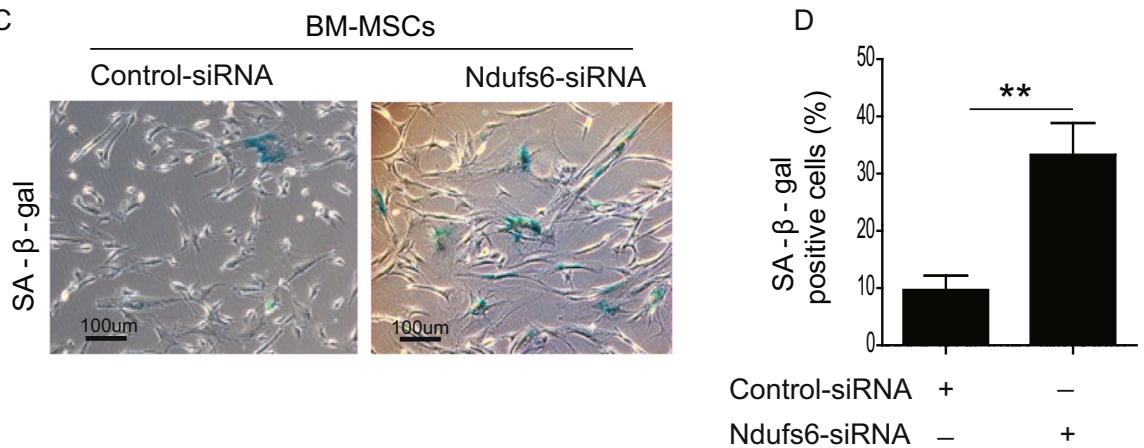

E

BM-MSCs
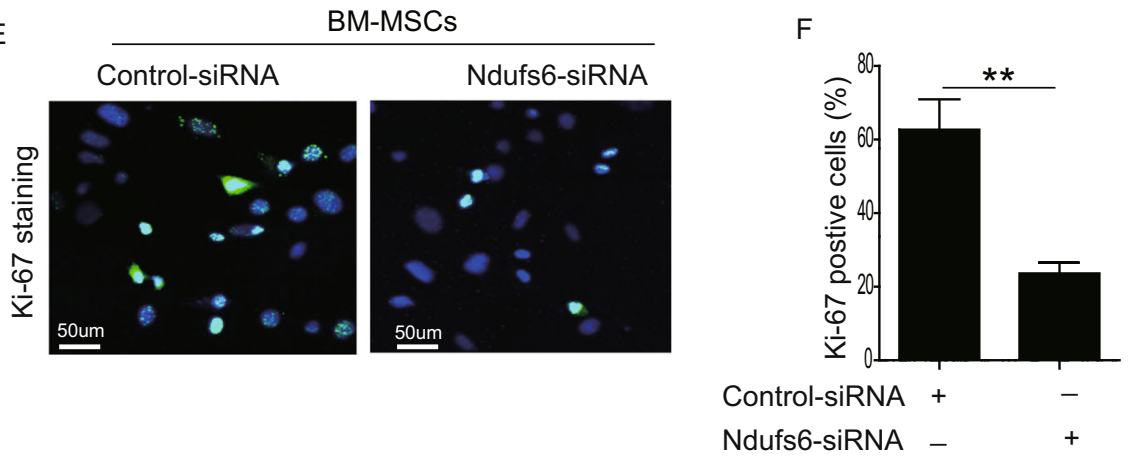

Fig. 3 Ndufs6 knockout accelerates cellular senescence in BM-MSCs. A The mRNA level of Ndufs6, p53, and p21 was detected in BM-MSCs and Ndufs6-siRNA-treated BM-MSCs. B The protein level of Ndufs6, p53, and p21 was detected in BM-MSCs and Ndufs6-siRNA-treated BM-MSCs. C SA$\beta$-gal staining showing $\beta$-gal-positive cells in BM-MSCs and Ndufs6-siRNA-treated BM-MSCs. D The $\beta$-gal positivity in BM-MSCs and Ndufs6-siRNAtreated BM-MSCs was quantified by calculating the ratio of stained cells to total cells. $\mathbf{E}$ The proliferation of BM-MSCs and Ndufs6-siRNA-treated BMMSCs was assessed by Ki-67 staining. F The Ki-67 positivity in BM-MSCs and Ndufs6-siRNA-treated BM-MSCs was quantified by calculating the ratio of stained cells to total cells. Results are presented as mean \pm SD. $n=3 .{ }^{* *} p<0.01 ;{ }^{* * *} p<0.001$. 


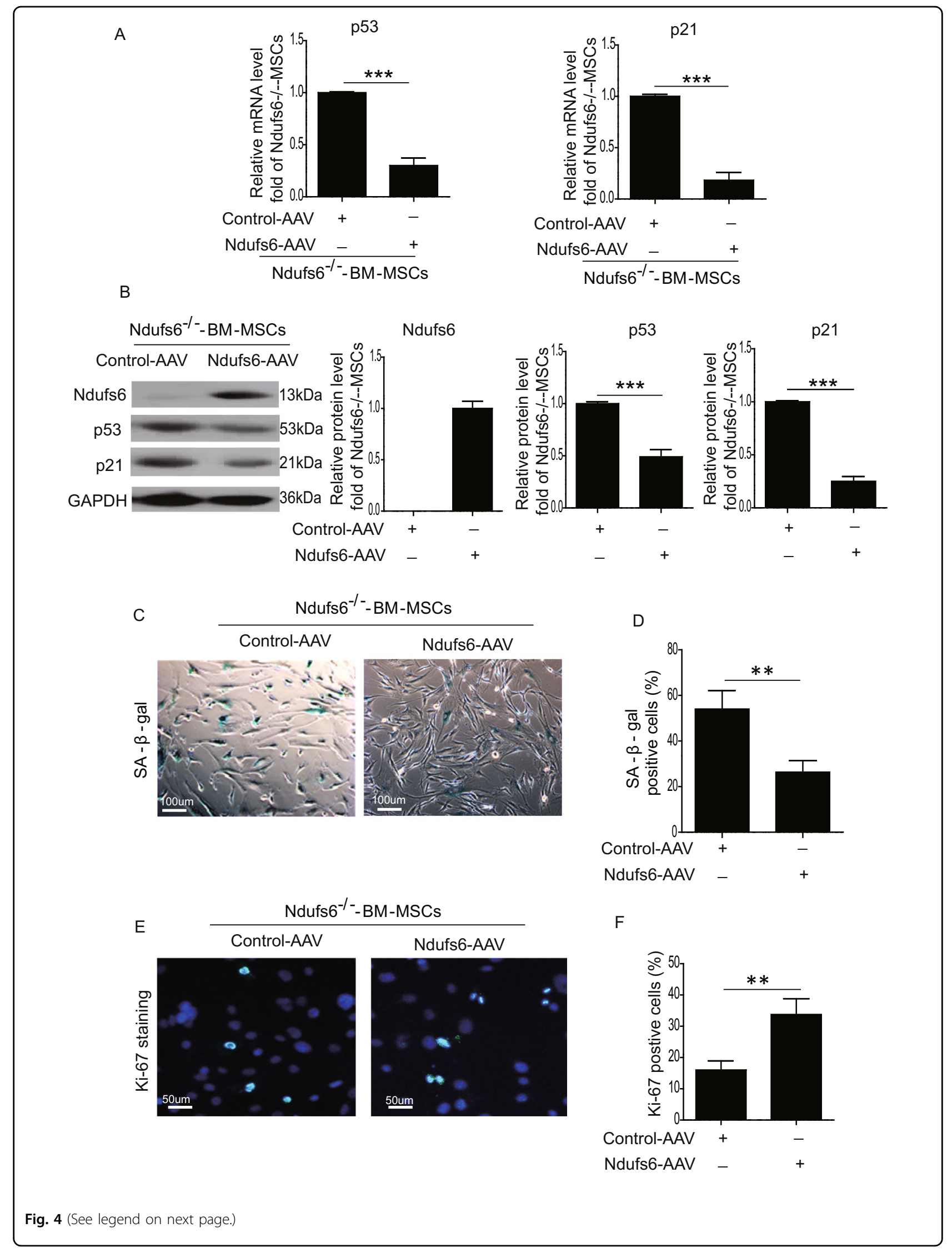


(see figure on previous page)

Fig. 4 Overexpression of Ndufs 6 in Ndufs $6^{-1-}$-BM-MSCs decreases cellular senescence. A The mRNA level of Ndufs6, p53, and p21 was detected by RT-PCR in Ndufs6 ${ }^{-1-}$-BM-MSCs and Ndufs6-AAV2-treated Ndufs6 ${ }^{-1-}$-BM-MSCs. B The protein level of Ndufs6, p53, and p21 was detected by western blotting in Ndufs $6^{-/-}$-BM-MSCs and Ndufs6-AAV-treated Ndufs6 ${ }^{-/}$BM-MSCs. C SA- $\beta$-gal staining showing $\beta$-gal-positive cells in Ndufs6 ${ }^{-1-}$ -BM-MSCs and Ndufs6-AAV-treated Ndufs $6^{-1-}$ BM-MSCs. D The $\beta$-gal positivity in Ndufs $6^{-1-}$-BM-MSCs and Ndufs6-AAV-treated Ndufs $6^{-1-}$ BM-MSCs was quantified by calculating the ratio of stained cells to total cells. $\mathbf{E}$ The proliferation of Ndufs $6^{-1-}$-BM-MSCs and Ndufs6-AAV-treated Ndufs6 ${ }^{-1-}$ BMMSCs was assessed by Ki-67 staining. $\mathbf{F}$ The Ki-67 positivity in Ndufs6 ${ }^{-1-}$-BM-MSCs and Ndufs6-AAV-treated Ndufs6 ${ }^{-1-}$ BM-MSCs was quantified by calculating the ratio of stained cells to total cells. Results are presented as mean \pm SD. $n=3 .{ }^{* *} p<0.01 ;{ }^{* * *} p<0.001$.

of MSC ageing, but also accelerates MSC ageing, thus downregulation of Ndufs6 and MSC ageing creates a vicious circle. Third, we established that depressed Ndufs6 leads to complex I functional deficiency, and activates excessive ROS and p53/p21, thus accelerating cellular senescence of MSCs. Fourth, inhibition of ROS or replenishment of Ndufs6 attenuates absence of Ndufs6induced cellular senescence.

Over the past decades, the senescence of MSCs has attracted huge attention due to their critical role in regenerative medicine. Senescent MSCs exhibit decrease differentiation capacity, anti-inflammation capacity, and reduced immunomodulatory activity ${ }^{30,32-34}$. Understanding the potential mechanisms that underlie MSC senescence is essential to the development of novel strategies to improve MSC quality, especially those from aged donors. It has been well documented that genetic alterations to MSCs contributes to cellular senescence. UBC knockdown induces senescence of MSCs and impairs their functions, whereas UBC overexpression ameliorates MSC senescence and improves their proliferative activity $^{35}$. Conditional knockdown of Foxp1 in MSCs leads to premature senescence as evidenced by impaired bone mass and reduced MSC self-renewal capacity ${ }^{36}$. Knockout of WT p53-inducible phosphatase-1 in BM-MSCs results in premature characteristics of senescence, including a typical senescent morphology, increased $\beta$-gal activity, and decreased proliferative capacity ${ }^{37}$. Given the known evidence that a genetic defect is closely linked to cellular senescence, we sought to determine whether Ndufs6 mediates senescence in MSCs. We found that the differentiation capacity was greatly reduced in Ndufs $6^{-1-}$-BMMSCs compared with WT MSCs. Moreover, Ndufs6 ${ }^{-1-}$ -BM-MSCs exhibited enhanced $\beta$-gal activity and reduced proliferative ability, suggesting that Ndufs6 may be involved in cellular senescence. Nonetheless it has yet to be shown how Ndufs6 mediates MSC senescence.

Complex I is the major carrier of electron and subsequent superoxide production in mitochondrial membrane $^{38}$. Complex I is the most vulnerable enzyme and the first to be damaged in many mitochondrial disorders. It has also been suggested that complex I malfunction is involved in the pathogenesis of diabetes and ageing. Nonetheless it is composed of at least 45 different subunits whose primary structures have only just been determined ${ }^{39}$. Understanding how the subunits of complex I contribute to stem cell ageing is just the beginning. Many subunits of complex I have been referred to as accessory (or supernumerary) subunits. Initially, these accessory subunits have been considered to be nonessential to the structure and function of mitochondrial complex I. Nonetheless recent research has questioned whether these "accessory subunits" are really accessory. Some of these subunits including Ndufs6 are absolutely essential for complex I function and loss or mutations of these subunits can lead to complex I defects. Mutations of Ndufa9, a Q-module subunit for complex I, cause mitochondrial complex I assembly defect, leading to a severe and fatal neonatal phenotype ${ }^{40}$. Mutations in Ndufb10, an accessory subunit of complex I, results into complex I deficiency, thus impairing the oxidation ${ }^{41}$. Conditional ablation of Ndufa5 induces partial complex I deficiency, leading to lethargy and loss of motor skills in mice $^{42}$. In the current study, following bioinformatics analysis that Ndufs6 downregulation is a hallmark of BMMSCs ageing, we verified that Ndufs6 is also an inducer of MSC ageing, closely associated with downregulated Ndufs6-provoked complex I deficiency.

Accumulating evidence has demonstrated that complex I deficiency can cause increased ROS generation, leading to several devastating mitochondrial disorders. Leman et al. analyzed several fibroblast cell lines isolated from patients with inherited complex I deficiency and found that ROS was greatly enhanced ${ }^{43}$. Knockout of Ndufs4-induced complex I deficiency triggers ROS generation and lipid droplet accumulation in glia, and subsequent neurodegenerative disease in mice ${ }^{44}$. Accordingly, we also observed elevated ROS in Ndufs6 ${ }^{-1-}$-BM-MSCs compared with BMMSCs. Further, Ndufs6-siRNA-treated BM-MSCs exhibited complex I deficiency and enhanced ROS, suggesting that complex I deficiency contributes to ROS generation. It is well established that ROS can trigger cellular senescence via regulation of multiple pathways. Inflammatory cytokine TNF- $\alpha$ can activate the ROS/NF- $k B$ pathway to induce nucleus pulposus cell senescence ${ }^{45}$. Iron overload-induced ROS accumulation induces BM-MSC senescence via upregulation of the protein expression of p53, ERK, and p38 (ref. $\left.{ }^{46}\right)$. Simvastatin treatment can induce senescence of human melanoma cells via activation of the ROS/p53/ 


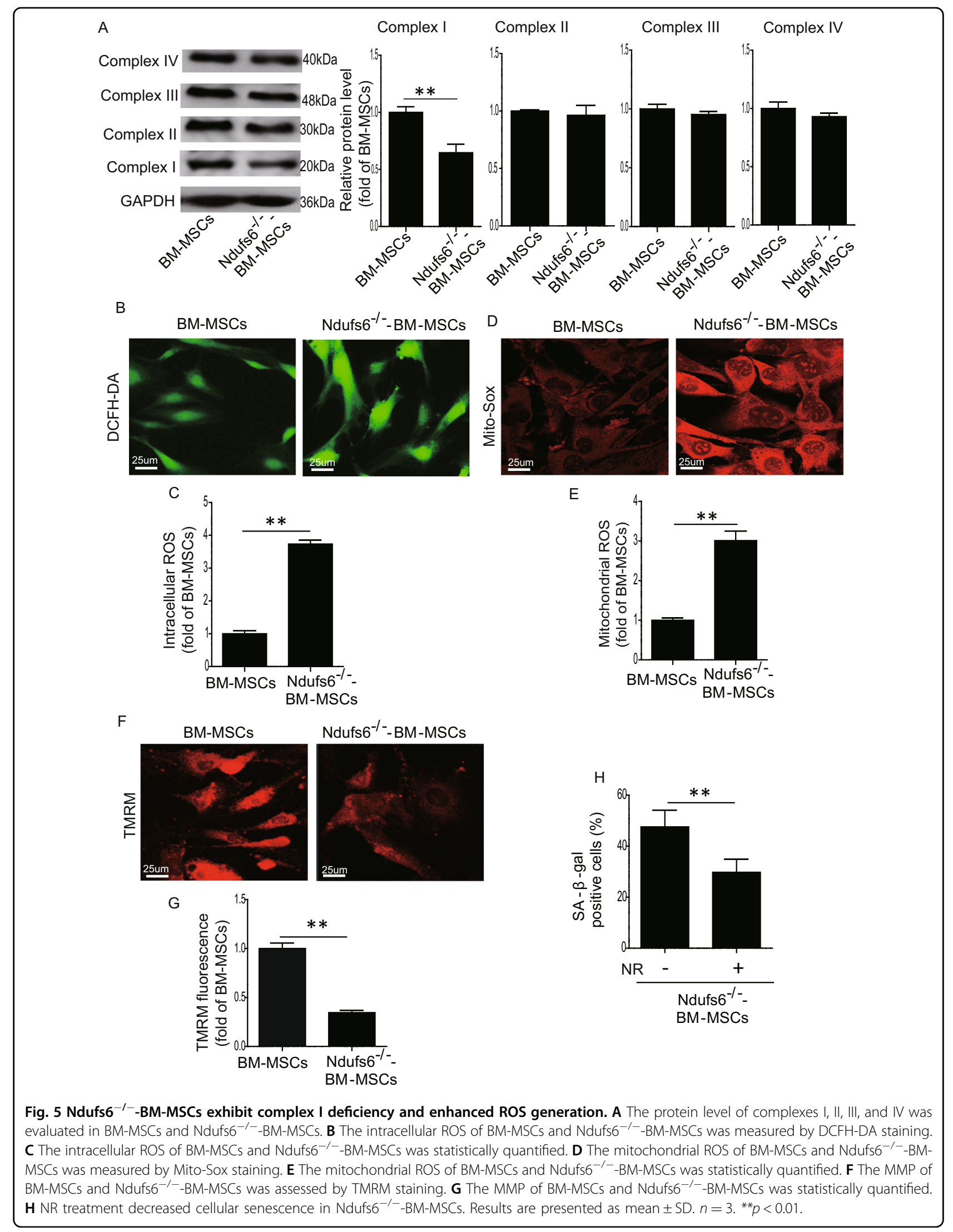




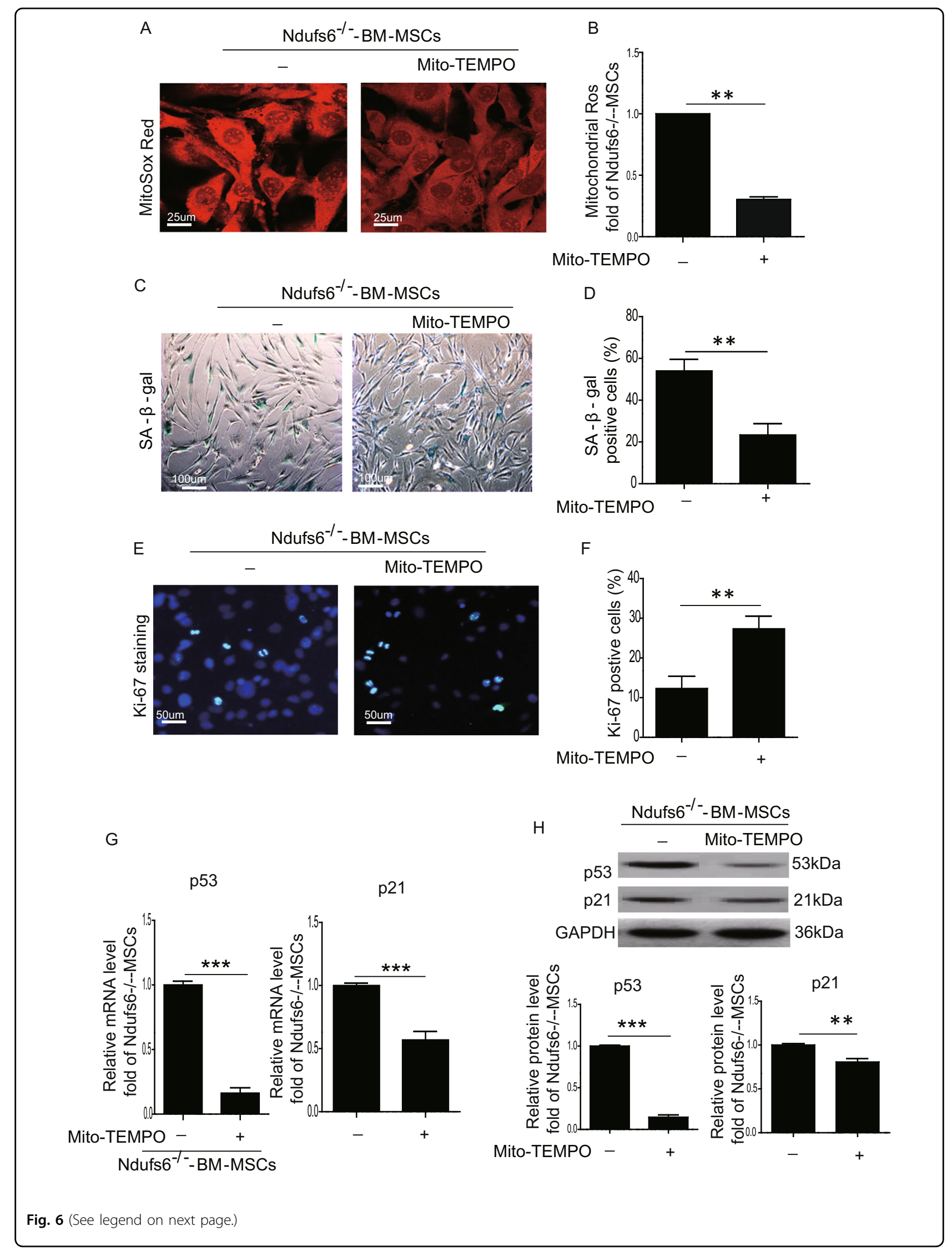


(see figure on previous page)

Fig. 6 Inhibition of ROS decreases cellular senescence of Ndufs6 ${ }^{-1-}$-BM-MSCs. A The mitochondrial ROS of Ndufs6 ${ }^{-1-}$-BM-MSCs and MitoTEMPO-treated Ndufs $6^{-1-}$-BM-MSCs was measured by Mito-Sox staining. B The mitochondrial ROS of Ndufs $6^{-1-}$-BM-MSCs and Mito-TEMPO-treated Ndufs $6^{-1-}$-BM-MSCs was statistically quantified. C SA- $\beta$-gal staining showing $\beta$-gal-positive cells in Ndufs $6^{-1-}$-BM-MSCs and Mito-TEMPO-treated

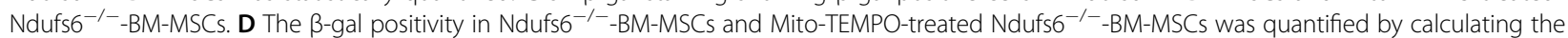
ratio of stained cells to total cells. $\mathbf{E}$ The proliferation of Ndufs $6^{-1-}$-BM-MSCs and Mito-TEMPO-treated Ndufs6 ${ }^{-1-}$-BM-MSCs was assessed by Ki-67 staining. $\mathbf{F}$ The Ki-67 positivity in Ndufs6 ${ }^{-1-}$-BM-MSCs and Mito-TEMPO-treated Ndufs $6^{-1-}$-BM-MSCs was quantified by calculating the ratio of stained cells to total cells. G The mRNA level of p53 and p21 was assessed in Ndufs6 ${ }^{-1-}$-BM-MSCs and Mito-TEMPO-treated Ndufs6 ${ }^{-1-}$-BM-MSCs. $\mathbf{H}$ The protein level of p53 and p21 was assessed in Ndufs6 ${ }^{-1-}$-BM-MSCs and Mito-TEMPO-treated Ndufs $6^{-1-}$-BM-MSCs. Results are presented as mean \pm SD. $n=3 .{ }^{* *} p<0.01$.

p21 signaling pathway ${ }^{47}$. In this study, we used MitoTEMPO to treat Ndufs $6^{-1-}$-BM-MSCs, and found that cellular senescence was greatly reduced along with a decreased level of ROS and protein level of p53/p21, indicating that the ROS/p53/p21 signaling pathway is involved in Ndufs6 knockout-induced cellular senescence of MSCs.

In summary, based on the above findings, we conclude that depressed Ndufs6 and MSC ageing interact in a manner of reciprocal causation. Ndufs6 defect impairs complex I function and activates the ROS/p53/ p21 signaling pathway, leading to cellular senescence of MSCs. Our study provides novel insight to precisely target the mitochondria subunit to prevent senescence of MSCs.

\section{Acknowledgements}

We thank Dr. David R. Thorburn (Murdoch Children's Research Institute, Australia) for Ndufs6 gt/gt mice. We thank reviewers for their helpful comments and Ms. Sarah Mercia Aglionby for English editing. This research was in part supported by National Natural Science Grant of China (No. 81700259 to Y.Z.; Nos 31571407 and 31270967 to Q.L.); Hong Kong Research Grant Council General Research Fund (HKU17113816 and HKU772510M to Q. L.); the Guangdong Provincial People's Hospital Grant for Talent Introduction (No. Y012018144 to Y.Z.), the High-level Hospital Construction Project of Guangdong Provincial People's Hospital (DFJH201918 to Y.Z.). General Research Fund of Research Grants Council of Hong Kong (No. 17117918 to B.Y.), and Seed Fund for Basic Research of University of Hong Kong (No. 201811159196 to B.Y.).

\section{Author details}

${ }^{1}$ Department of Emergency Medicine, Guangdong Provincial People's Hospital, Guangdong Academy of Medical Sciences, Guangzhou, Guangdong, China. ${ }^{2}$ Guangzhou Women and Children's Medical Centre, Guangzhou Medical University, Guangzhou, Guangdong, China. ${ }^{3}$ Department of Medicine, Li Ka Shing Faculty of Medicine, the University of Hong Kong, Hong Kong SAR, China. ${ }^{4}$ Clinical Translational Medical Research Center, Shanghai East Hospital, Tongji University School of Medicine, Shanghai, China. ${ }^{5}$ Guangdong Cardiovascular Institute, Guangdong Provincial People's Hospital, Guangdong Academy of Medical Sciences, Guangzhou, Guangdong, China. ${ }^{6}$ Department of Computer Science, Faculty of Engineering, the University of Hong Kong, Hong Kong SAR, China. ${ }^{7}$ The State Key Laboratory of Pharmaceutical Biotechnology, the University of Hong Kong, Hong Kong SAR, China

\section{Author contributions}

Q.L. supervised the whole project. Y.Z. and Q.L. designed the experiment and wrote the manuscript. Y.Z., L.G., S.H., L.C., C. L, J.Z., Y.H., X. Zhang, X. Zhou, X. Liang and D.J. performed the majority of the experiments and collected data. J.Q., J.Z., X. Li, S.Z., and H.-F.T. helped to analyze the data and provided the materials. C. Liao and Y.B. analyzed the bioinformatics data. All authors read and approved the final version of the manuscript.

\section{Conflict of interest}

The authors declare that they have no conflict of interest.

\section{Publisher's note}

Springer Nature remains neutral with regard to jurisdictional claims in published maps and institutional affiliations.

Supplementary Information accompanies this paper at (https://doi.org/ 10.1038/s41419-020-03289-w).

Received: 8 May 2020 Accepted: 25 November 2020

Published online: 15 December 2020

\section{References}

1. Zhang, Y. et al. iPSC-MSCs with high intrinsic MIRO1 and sensitivity to TNFalpha yield efficacious mitochondrial transfer to rescue anthracycline-induced cardiomyopathy. Stem Cell Rep. 7, 749-763 (2016).

2. Natsumeda, M. et al. A combination of allogeneic stem cells promotes cardiac regeneration. J. Am. Coll. Cardiol. 70, 2504-2515 (2017).

3. Li, X. et al. Mitochondrial transfer of induced pluripotent stem cellderived mesenchymal stem cells to airway epithelial cells attenuates cigarette smoke-induced damage. Am. J. Respir. Cell Mol. Biol. 51, 455-465 (2014).

4. Butler, J. et al. Intravenous allogeneic mesenchymal stem cells for nonischemic cardiomyopathy: safety and efficacy results of a phase I-A randomized trial. Circ. Res. 120, 332-340 (2017).

5. Zhang, Y. et al. Macrophage migration inhibitory factor rejuvenates aged human mesenchymal stem cells and improves myocardial repair. Aging 11, 12641-12660 (2019)

6. Shang, J. et al. miR-29c-3p promotes senescence of human mesenchymal stem cells by targeting CNOT6 through p53-p21 and p16-pRB pathways. Biochim. Biophys. Acta 520-532, 2016 (1863).

7. Liu, M. et al. Adipose-derived mesenchymal stem cells from the elderly exhibit decreased migration and differentiation abilities with senescent properties. Cell Transpl. 26, 1505-1519 (2017).

8. Okada, M. et al. Abrogation of age-induced MicroRNA-195 rejuvenates the senescent mesenchymal stem cells by reactivating telomerase. Stem Cells $\mathbf{3 4}$, 148-159 (2016)

9. Ma, Y. et al. Autophagy controls mesenchymal stem cell properties and senescence during bone aging. Aging Cell 17, e12709 (2018).

10. Khrapko, K. \& Vijg, J. Mitochondrial DNA mutations and aging: devils in the details? Trends Genet. 25, 91-98 (2009).

11. Wiley, C. D. et al. Mitochondrial dysfunction induces senescence with a distinct secretory phenotype. Cell Metab. 23, 303-314 (2016).

12. Garcia-Prat, L. et al. Autophagy maintains stemness by preventing senescence. Nature 529, 37-42 (2016).

13. Sun, N., Youle, R. J. \& Finkel, T. The mitochondrial basis of aging. Mol. Cell 61, 654-666 (2016).

14. Stab, B. R. II et al. Mitochondrial functional changes characterization in young and senescent human adipose derived MSCs. Front Aging Neurosci. 8, 299 (2016).

15. Onodera, Y. et al. miR-155 induces ROS generation through downregulation of antioxidation-related genes in mesenchymal stem cells. Aging Cell 16, 1369-1380 (2017). 
16. Zhang, M. et al. Cholesterol retards senescence in bone marrow mesenchymal stem cells by modulating autophagy and ROS/p53/p21(Cip1/Naf1) pathway. Oxid. Med. Cell Longev. 2016, 7524308 (2016).

17. Kizilay Mancini, O. et al. Mitochondrial oxidative stress reduces the immunopotency of mesenchymal stromal cells in adults with coronary artery disease. Circ. Res. 122, 255-266 (2018).

18. Wang, Y. \& Hekimi, S. Mitochondrial dysfunction and longevity in animals: untangling the knot. Science 350, 1204-1207 (2015).

19. Yu, D. M. et al. Caveolin-1 deficiency induces premature senescence with mitochondrial dysfunction. Aging Cell 16, 773-784 (2017).

20. Koch, L. Clinical genetics: mitochondrial replacement techniques under the spotlight. Nat. Rev. Genet. 15, 516 (2014).

21. Zhang, $H$. et al. $N A D(+)$ repletion improves mitochondrial and stem cell function and enhances life span in mice. Science 352, 1436-1443 (2016).

22. Schapira, A. H. Mitochondrial disease. Lancet 368, 70-82 (2006).

23. Forbes, J. M. et al. Deficiency in mitochondrial complex I activity due to Ndufs6 gene trap insertion induces renal disease. Antioxid. Redox Signal 19, 331-343 (2013).

24. Leipnitz, G. et al. Evaluation of mitochondrial bioenergetics, dynamics, endoplasmic reticulum-mitochondria crosstalk, and reactive oxygen species in fibroblasts from patients with complex I deficiency. Sci. Rep. 8, 1165 (2018).

25. Nouws, J., Te Brinke, H., Nijtmans, L. G. \& Houten, S. M. ACAD9, a complex I assembly factor with a moonlighting function in fatty acid oxidation deficiencies. Hum. Mol. Genet 23, 1311-1319 (2014).

26. Kirby, D. M. et al. NDUFS6 mutations are a novel cause of lethal neonatal mitochondrial complex I deficiency. J. Clin. Investig. 114, 837-845 (2004).

27. Ke, B. X. et al. Tissue-specific splicing of an Ndufs6 gene-trap insertion generates a mitochondrial complex I deficiency-specific cardiomyopathy. Proc. Natl Acad. Sci. USA 109, 6165-6170 (2012)

28. Zhang, $Y$. et al. Absence of NUCKS augments paracrine effects of mesenchymal stem cells-mediated cardiac protection. Exp. Cell Res. 356, 74-84 (2017)

29. Liang, X. et al. Activation of NRG1-ERBB4 signaling potentiates mesenchymal stem cell-mediated myocardial repairs following myocardial infarction. Cell Death Dis. 6, e1765 (2015).

30. Huang, $X$. et al. Adipose-derived mesenchymal stem cells isolated from patients with abdominal aortic aneurysm exhibit senescence phenomena. Oxid. Med. Cell Longev. 2019, 1305049 (2019).

31. Zhang, Z. et al. PSCs Reveal PUFA-provoked mitochondrial stress as a central node potentiating RPE degeneration in Bietti's crystalline dystrophy. Mol Ther. 28, 2642-2661 https://doi.org/10.1016/j.ymthe.2020.07.024 (2020).
32. Kapetanou, M., Chondrogianni, N., Petrakis, S., Koliakos, G. \& Gonos, E. S. Proteasome activation enhances stemness and lifespan of human mesenchymal stem cells. Free Radic. Biol. Med. 103, 226-235 (2017).

33. Bustos, M. L. et al. Aging mesenchymal stem cells fail to protect because of impaired migration and antiinflammatory response. Am. J. Respir. Crit. Care Med. 189, 787-798 (2014).

34. Sepulveda, J. C. et al. Cell senescence abrogates the therapeutic potential of human mesenchymal stem cells in the lethal endotoxemia model. Stem Cells 32, 1865-1877 (2014).

35. Kim, J. et al. Ubiquitin $C$ decrement plays a pivotal role in replicative senescence of bone marrow mesenchymal stromal cells. Cell Death Dis. 9, 139 (2018).

36. Li, $\mathrm{H}$. et al. FOXP1 controls mesenchymal stem cell commitment and senescence during skeletal aging. J. Clin. Investig. 127, 1241-1253 (2017).

37. Tang, $Y$. et al. Wipl knockout inhibits the proliferation and enhances the migration of bone marrow mesenchymal stem cells. Exp. Cell Res. 334 310-322 (2015).

38. Lenaz, G. et al. Mitochondrial Complex I: structural and functional aspects. Biochim. Biophys. Acta 1757, 1406-1420 (2006).

39. Zhu, J., Vinothkumar, K. R. \& Hirst, J. Structure of mammalian respiratory complex I. Nature 536, 354-358 (2016).

40. Baertling, F. et al. NDUFA9 point mutations cause a variable mitochondrial complex I assembly defect. Clin. Genet. 93, 111-118 (2018).

41. Friederich, M. W. et al. Mutations in the accessory subunit NDUFB10 result in isolated complex I deficiency and illustrate the critical role of intermembrane space import for complex I holoenzyme assembly. Hum. Mol. Genet. 26, 702-716 (2017).

42. Peralta, S. et al. Partial complex I deficiency due to the CNS conditional ablation of Ndufa5 results in a mild chronic encephalopathy but no increase in oxidative damage. Hum. Mol. Genet. 23, 1399-1412 (2014).

43. Leman, G. et al. Assembly defects induce oxidative stress in inherited mitochondrial complex I deficiency. Int. J. Biochem. Cell Biol. 65, 91-103 (2015).

44. Liu, L. et al. Glial lipid droplets and ROS induced by mitochondrial defects promote neurodegeneration. Cell 160, 177-190 (2015).

45. Xie, J. et al. Osteogenic protein-1 attenuates the inflammatory cytokineinduced NP cell senescence through regulating the ROS/NF-kappaB pathway. Biomed. Pharmacother. 99, 431-437 (2018).

46. Yang, F. et al. Melatonin protects bone marrow mesenchymal stem cells against iron overload-induced aberrant differentiation and senescence. $J$. Pineal. Res. 63, e12422 (2017).

47. Guterres, F. A., Martinez, G. R., Rocha, M. E. \& Winnischofer, S. M. Simvastatin rises reactive oxygen species levels and induces senescence in human melanoma cells by activation of p53/p21 pathway. Exp. Cell Res. 319, 2977-2988 (2013). 